\begin{abstract}
Alexandre S. Pyrrho - Henrique Leonel Lenzi
Juliene Antonio Ramos · Roberto Moura-Neto

Fabio Christiane O. F. Cachem · Célia Santos da

Silva - Christina Maeda Takiya - Cerli Rocha Gattass

\section{Dexamethasone treatment improves morphological and hematological parameters in chronic experimental schistosomiasis}

Received: 12 January 2004 / Accepted: 21 January 2004 / Published online: 4 March 2004

(C) Springer-Verlag 2004

\begin{abstract}
Schistosomiasis, a chronic disease with considerable social impact, is an important health problem in many countries. To investigate the possible use of immunomodulators as coadjuvants in the treatment of chronic Schistosoma mansoni infection, we evaluated the effect of dexamethasone on histological, hematological, and biochemical parameters that reflect disease severity and morbidity. Animals treated from the first day or after 35 days of infection, were analyzed. In both groups, dexamethasone: (1) induced a decrease in the number of granulomas in hepatic tissue without affecting the alanine aminotransferase profile, (2) reduced splenomegaly and hepatomegaly associated with disease, and (3) improved hemoglobin concentration, hematocrit values and reduced the percentage of reticulocytes, preventing the development of anemia that occurs in the chronic phase of infection. These data suggest that treatment with dexamethasone results in a mild course of murine schistosomiasis and point to this drug as a promising agent to complement $S$. mansoni specific treatment.
\end{abstract}

A. S. Pyrrho $(\bowtie) \cdot$ J. A. Ramos · R. Moura-Neto

F. C. O. F. Cachem - C. Santos da Silva

Departamento de Análises Clínicas e Toxicológicas,

Faculdade de Farmácia, Cidade Universitária,

CCS, UFRJ, 21949-900 Rio de Janeiro, RJ, Brazil

E-mail: pyrrho@pharma.ufrj.br

Fax: + 55-21-25626423

A. S. Pyrrho · C. R. Gattass

Instituto de Biofísica Carlos Chagas Filho,

Cidade Universitária, UFRJ, 21949-900

Rio de Janeiro, RJ, Brazil

H. L. Lenzi

Departamento de Patologia,

Instituto Oswaldo Cruz, FIOCRUZ,

21040-360 Rio de Janeiro, RJ, Brazil

C. M. Takiya

Departamento de Histologia e Embriologia - Instituto de

Ciências Biomédicas, Cidade Universitária,

UFRJ, 21949-900 Rio de Janeiro, RJ, Brazil

\section{Introduction}

Schistosomiasis is a parasitic disease of great social impact due to its morbidity and consequent work disability (World Health Organisation 1997; Ross et al. 2002). Although morbidity is frequently associated with granuloma formation and subsequent liver fibrosis, the drugs used to treat this disease are generally unable to reduce the pathological sequelae of the chronic phase. Therefore, the search for new strategies able to reduce granuloma formation or its sequelae is highly relevant for disease treatment.

During the chronic phase of infection, the host presents alterations in hematological (eosinophilia, neutropenia, and anemia) and visceral (splenomegaly and hepatomegaly) parameters, which have often been correlated with morbidity (Fanning et al. 1981). Considering these alterations, Henderson et al. (1993) described two distinct syndromes in experimental chronic schistosomiasis: the hypersplenomegaly syndrome (HSS) characterized by massive splenomegaly, severe anemia, cachexia and high morbidity, and the moderate splenomegaly syndrome (MSS) with mild alterations and reduced mortality. Therefore, despite modern techniques, the measurement of these parameters is still of great relevance for the clinical evaluation of the disease.

Although much is known about schistosomiasis, many aspects of its pathogenesis remain unclear (Hagan et al. 1998). In addition to helping to elucidate these aspects, experimental models are crucial for the development of new drugs and vaccines (Cheever et al. 2002; Druilhe et al. 2002). In view of the immunomodulatory properties of dexamethasone, we have been using an experimental model of schistosomiasis to investigate the effects of this drug on disease severity and morbidity. In a previous paper, we showed that dexamethasone treatment caused a decrease in granuloma size and a reduction in periovular fibrosis deposition. Moreover, dexamethasone did not affect 
the parasite burden or oviposition capacity but caused a decrease in hepatic egg deposition (Pyrrho et al. 2002). To better understand the role of dexamethasone on disease severity, we investigated its effects on hematological and visceral parameters, hepatic enzyme profile, and the density of hepatic granulomas. The data show a decrease in the density of hepatic granulomas and a reduction in hepatosplenomegaly, anemia, neutropenia, and eosinophilia, without any increase in hepatic enzymes. These results are in agreement with our previous data and indicate that dexamethasone treatment minimizes the deleterious effects of experimental Schistosoma mansoni infection.

\section{Materials and methods}

Animals, the drug and infection

Adult C57BL/6J female mice (7-8 weeks old) were infected with 45 cercariae of the $\mathrm{BH}$ strain of $S$. mansoni, by the cutaneous route. Dexamethasone (Decadron, Prodome, Brazil), $1 \mathrm{mg} \mathrm{kg}$ body weight, was administered intramuscularly three times a week, until the end of the experiment (55th or 120th day postinfection-dpi). Control (N) and infected (I) animals were divided in four groups: non-treated ( $\mathrm{N}$ and $\mathrm{I})$, treated with saline $(\mathrm{N}+\mathrm{S}$ and $\mathrm{I}+\mathrm{S})$, treated with dexamethasone $\left(1 \mathrm{mg} \mathrm{kg}{ }^{-1}\right)$ from the beginning of the experiment $(\mathrm{N}+\operatorname{Dex} 0$ and $\mathrm{I}+\operatorname{Dex} 0)$ or 35 days after the beginning of the experiment $(\mathrm{N}+\operatorname{Dex} 35$ and $\mathrm{I}+$ Dex35). Animals from all groups (at least ten per group) were killed under anesthesia on the 55th or 120th dpi. During this time, they were maintained in controlled temperature and light conditions, fed a balanced diet and given sterile water ad libitum.

Hepatic granuloma density

Transverse sections of all liver lobes were collected, fixed in $4 \%$ buffered formaldehyde solution and embedded in paraffin. Sections of $5 \mu \mathrm{m}$ were stained with phosphomolibidic acid-picro-sirius red staining (PMA-PSR) (Dolber and Spach 1993). For the evaluation of granuloma density, stained slides were observed using bright field microscopy and all granulomas containing central viable eggs were quantified. All evaluations were performed blind by two different observers. The results were expressed as number of granulomas $/ \mathrm{mm}^{2}$.

Hematological data/liver and spleen weight

Blood was collected by axillary plexus incision in an EDTA (Merck, Germany) coated vial. Hemogram values were determined in a CC550-CELM (Companhia Equipadora de Laboratórios Modernos, São Paulo). Thin blood slides stained with Giemsa/ Wright (Merck, Germany) were used for differential leukocyte counts. Reticulocyte counts were performed using cresyl blue brilliant stain $(1 \%$ cresyl blue brilliant solution in $0.85 \%$ sodium chloride). After the mice were killed, the liver and spleen were removed and their weights determined.

\section{Alanine aminotransferase}

The serum alanine aminotransferase (ALT) levels, a marker of hepatocellular damage, were established by colorimetric assay using a commercial kit from Chiron Diagnostics (East Walple, Mass.) at 55 and $120 \mathrm{dpi}$.
Statistical analysis

Statistical analysis was performed with SigmaPlot for Windows software, version 5.0 (SPSS). The comparison between groups was done by non-paired Student's $t$-test. Values of $P<0.05$ were considered significant. Means $\pm \mathrm{SD}$ are given.

\section{Results}

\section{Hepatic granuloma density}

Independent of the treatment schedule used (I + Dex 0 or I + Dex35), animals treated with dexamethasone showed a decrease in the density of hepatic granuloma when compared with non-treated ones (Fig. 1). However, a significant reduction in granuloma density $(P<0.04)$ was only observed in the group that started receiving dexamethasone after the 35th dpi (I+Dex35) (Fig. 1). This observation is in agreement with previous results showing a decrease in the amount of eggs retained in the liver after dexamethasone treatment (Pyrrho et al. 2002).

\section{Hematological data/liver and spleen weight}

Alterations in hematocrit and variations in liver/spleen weight have been used by several researchers as indicators of disease severity in schistosomiasis (Fanning et al. 1981; Henderson et al. 1993; Adewusi et al. 1996; Eberl et al. 2001). We have shown that, independent of the treatment schedule used (I+Dex0 or I + Dex35), at the chronic phase of infection (120 dpi), the weights of the liver and spleen of infected treated animals were significantly lower than those of the controls (Fig. 2A, B).

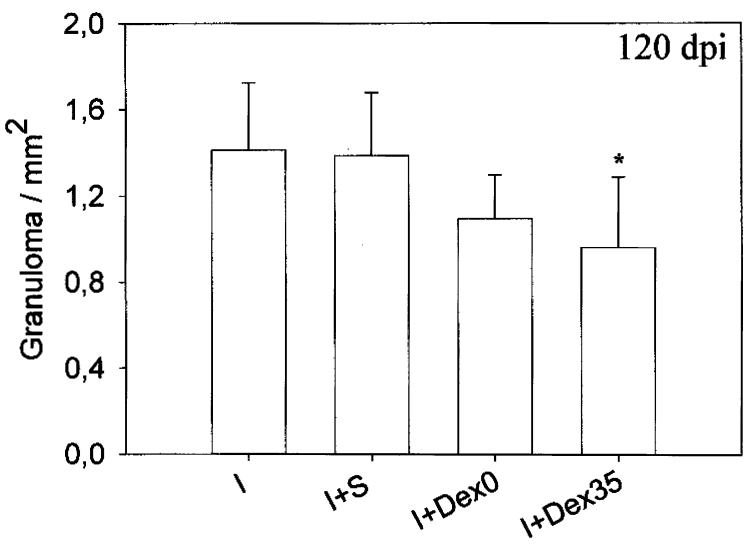

Fig. 1 Quantification of granuloma density in hepatic tissue Groups of mice were infected with 45 cercarieae/animal and left untreated or treated with saline $(S)$ or dexamethasone $\left(1 \mathrm{mg} \mathrm{kg}^{-1}\right)$ from the beginning of the experiment $(I+$ Dex 0$)$ or 35 days after the infection $(I+D e x 35)$. At 120 days post-infection the animals were killed and histological sections $(5 \mu \mathrm{m})$ of hepatic tissue were stained with phosphomolibidic acid-picro-sirius red (PMA-PSR). All granulomas containing a central viable egg were counted and the results were expressed as the mean $\pm \mathrm{SD}$ of granulomas $/ \mathrm{mm}^{2}$ (* indicates $P<0.039$ ) 

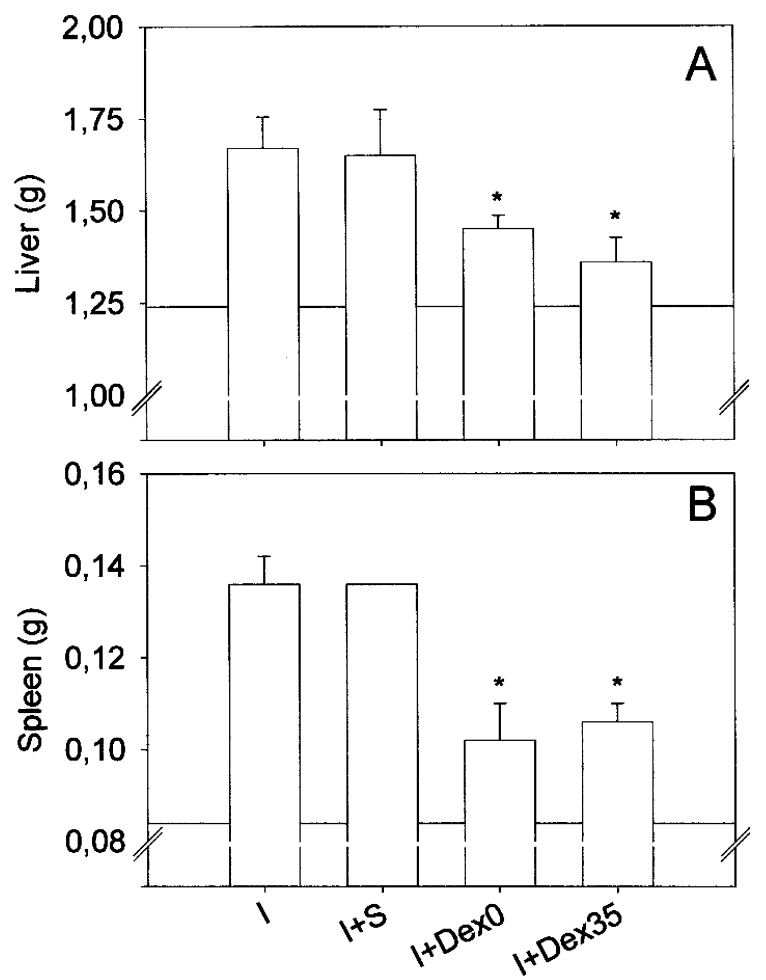

Fig. 2A, B Effects of dexamethasone on liver and spleen weight. Mice infected and treated as described in Fig. 1 were killed at 120 days post-infection and their livers (A) and spleens (B) were weighted. Results are expressed as mean $\pm \mathrm{SD}$ of at least ten animals. The baseline represents the mean values of liver and spleen weights of non-infected animals (* indicates $P<0.05$ )

Dexamethasone had no significant effect on non-infected mice submitted to the same schedule of treatment (baseline in Fig. 2).

Infected animals developed light anemia, expressed by lower levels of hemoglobin $(12.04 \pm 1.87)$ and hematocrit $(35.99 \pm 5.55)$ values than those observed in non-infected ones $(14.14 \pm 0.72$ and $43.90 \pm 4.61$, respectively). Treatment with dexamethasone partially prevented the anemia resulting from infection, causing a small improvement in hemoglobin concentration $(\mathrm{I}+\operatorname{Dex} 0=12.95 \pm 1.84 ; \quad \mathrm{I}+\operatorname{Dex} 35=12.21 \pm 1.29 \mathrm{SD})$ and hematocrit values $(\mathrm{I}+\operatorname{Dex} 0=39.54 \pm 5.53$; $\mathrm{I}+\operatorname{Dex} 35=39.63 \pm 7.62$ ). These effects were accompanied by a significant reduction in the percentage of reticulocytes in the treated animals (Fig. 3). In addition, treated infected animals also showed a reversion of the neutropenia (Fig. 4A) and a decrease in the eosinophilia provoked by infection (Fig. 4B).

\section{Alanine aminotransferase}

To investigate whether dexamethasone interferes with hepatotoxicity induced by infection, we evaluated the levels of ALT in the serum of infected-treated animals. As shown in Fig. 5, the serum levels of ALT were not affected by treatment.

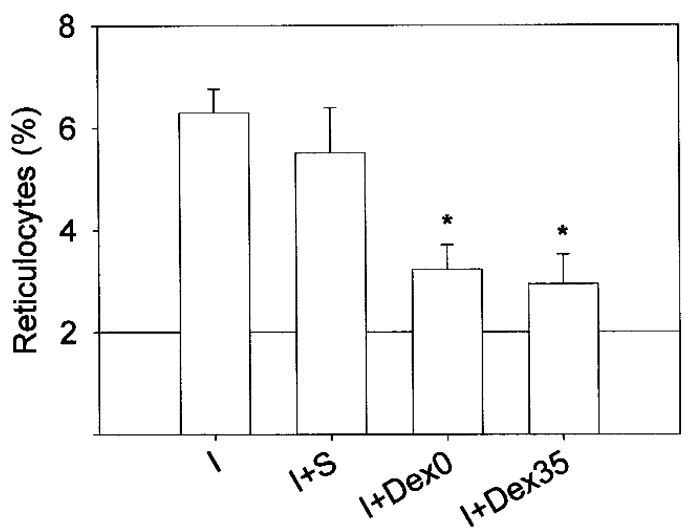

Fig. 3 Effects of dexamethasone on reticulocyte count. At 120 days after infection, blood of groups of mice treated as described in Fig. 1 was collected and used for reticulocyte evaluation. Results were expressed as mean \pm SD. The baseline represents mean values of non-infected animals. Statistical analyses were performed between infected non-treated $(I)$ and treated $(I+\operatorname{Dex} 0$ and $I+$ Dex35) groups (* indicates $P<0.001$ )

\section{Discussion}

In a previous paper, we showed that dexamethasone did not interfere with the mortality rate or parasite burden
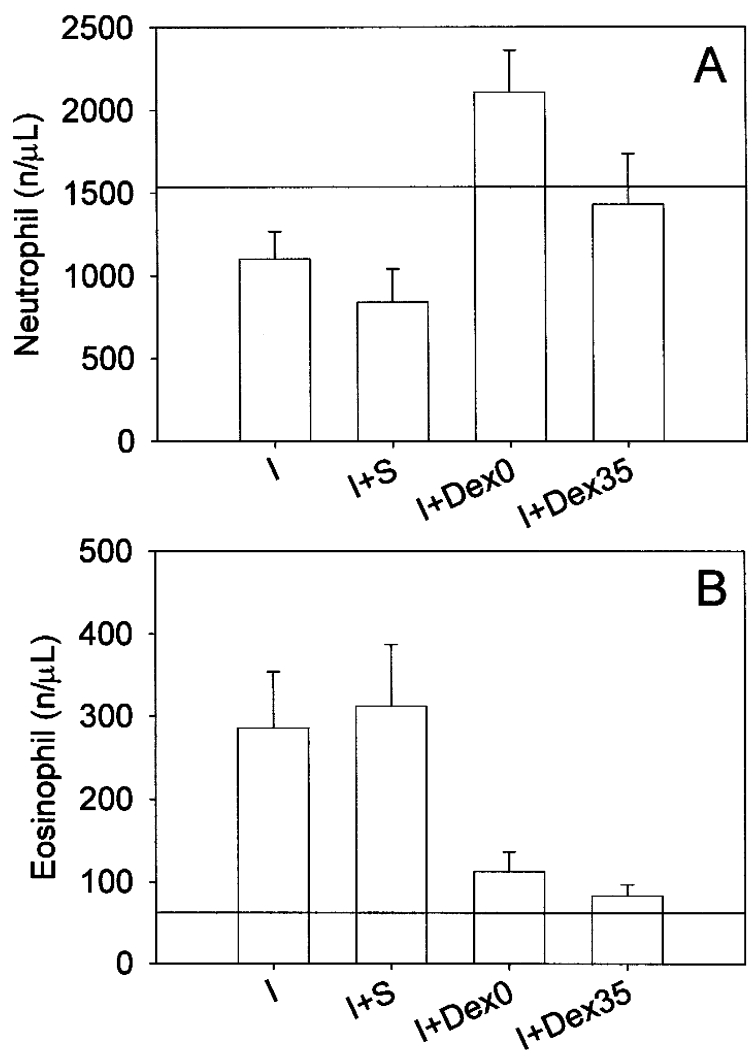

Fig. 4 Dexamethasone reverted neutropenia and eosinophilia in Schistosoma mansoni infection. Blood from the same groups of animals used in Fig. 3 was used for neutrophil and eosinophil counts. The absolute number of leukocytes was obtained by multiplication of specific percentage values by total leukocytes count. The baseline represents values of the non-infected group 


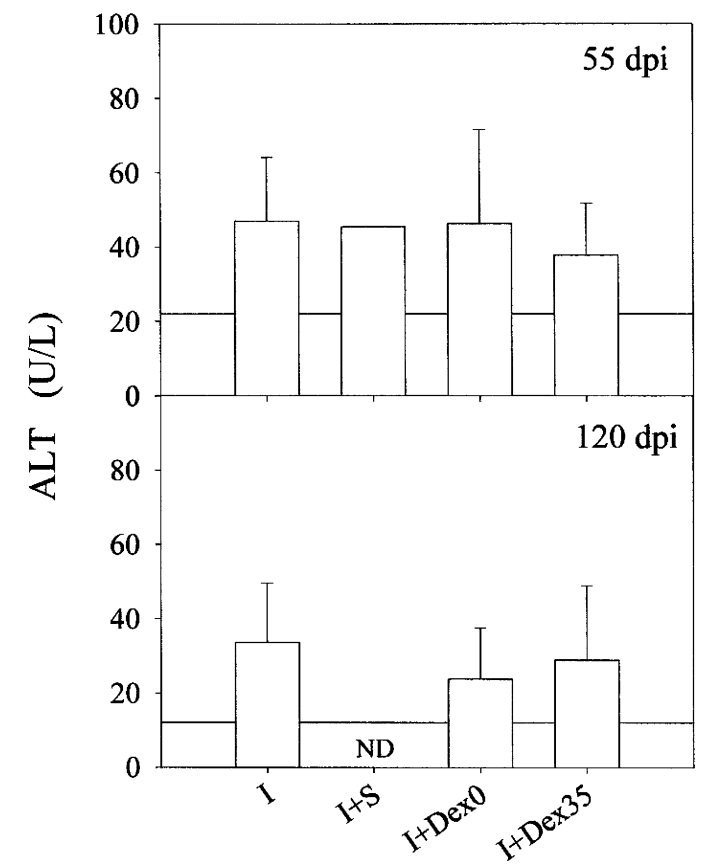

Fig. 5 Dexamethasone did not change the serum levels of ALT in $S$. mansoni infection. Sera from infected and treated groups of animals were collected at 55 and 120 days post-infection and measurements of ALT levels were performed by colorimetric assay. Results were expressed as mean $\pm \mathrm{SD}$. $N D$ Not done

in mice chronically infected with $S$. mansoni, but reduced granuloma size and fibrosis, and led to a modulation of cytokine production (Pyrrho et al. 2002). We demonstrate here that treatment with dexamethasone also leads to an improvement in parameters indicative of a decrease in disease severity, such as hepatosplenamegaly, hematological alterations, and the density of hepatic granuloma.

The main factors involved in the pathogenesis of schistosomiasis are eggs trapped in hepatic tissue and the host response elicited by them (Warren et al. 1967; Cheever et al. 1998). According to Cheever et al. (1983, 1987), a decrease in granuloma number and in the consequent amount of fibrosis results in low portal hypertension and therefore a mild form of the disease. As shown in Fig. 1, dexamethasone treated animals have a significant reduction in the density of hepatic granulomas, indicating a beneficial effect of dexamethasone treatment on infected hosts. Interestingly, this reduction was greater in the I+ Dex35 group, indicating a possible therapeutic application of dexamethasone in patients in the acute phase of schistosomiasis, as already proposed by Lambertucci et al. (1989).

In addition to its effect on hepatic granuloma density, dexamethasone treatment reduced the number of peripheral eosinophils and brought peripheral neutrophils into a normal range, reverting the eosinophilia and neutropenia characteristic of the chronic phase of schistosomiasis. This neutropenia may be partially due to a delay in the maturation of neutrophils in the bone marrow and spleen caused by a specific inhibitory activity demonstrated in the sera of chronic patients (Borojevic et al. 1983) and in experimental schistosomiasis (Santos-Da-Silva et al. 1988). The reversion of neutropenia may decrease susceptibility to bacterial and fungal infections associated with chronic schistosomiasis (Andrade and Bina 1983; Lambertucci et al. 2001). Therefore, it is possible that the alteration in peripheral leucocytes induced by dexamethasone may reduce granuloma cell recruitment, explaining the decrease in granuloma size observed in dexamethasone treated animals (Pyrrho et al. 2002), without affecting hepatotoxicity as shown by the lack of alterations in ALT levels (Fig. 5).

The reduction in hepatomegaly may be due to a decrease in the number of liver granulomas, probably reflecting less hepatic involvement and portal hypertension. The amelioration in hepatic fibrosis reported previously (Pyrrho et al. 2002) is in agreement with this hypothesis. Splenomegaly is also an important parameter in schistosomiasis due to its correlation with an increase in morbidity (Henderson et al. 1993; Bosshardt et al. 1997). It is attributed to chronic congestion caused by portal hypertension and hyperplasia of the reticuloendothelial cells (Andrade 1962; Bagshawe 1970). The reduction in splenomegaly in dexamethasone-treated animals (Fig. 2) can probably be associated with a decrease in both of these pathogenic factors. Therefore, the ability of dexamethasone to modulate hepato- and splenomegaly indicates its capacity to alter disease development towards a milder course.

The beneficial effect of dexamethasone in schistosomiasis was reinforced by the presence of normal hemoglobin rates and the absence of reticulocytosis (Fig. 3A, C) in treated animals, demonstrating the capacity of the drug to prevent the development of anemia, a parameter generally correlated with disease morbidity (Fanning et al. 1981; Henderson et al. 1993). The development of anemia in schistosomiasis has been attributed to many causes including gastrointestinal hemorrhages, hemodilution and hypersplenism (Jamra et al. 1964). If, as proposed elsewhere (Woodruff et al. 1966), there is a correlation between splenomegaly or hypersplenism and anemia, then the effect of dexamethasone in preventing anemia may be partially explained by the decrease in splenomegaly. Since anemia can involve many physiological functions, its prevention would improve the clinical condition of the animal by reducing morbidity due to parasite infections (Guyatt et al. 2001), including schistosomiasis.

The drugs currently used for schistosomiasis treatment lead to parasite elimination and also to a slight reduction in hepatic fibrosis (Bina and Prata 1983; Homeida et al. 1991). However, in spite of treatment, part of the population remains infected (Utzinger et al. 2000; Garba et al. 2001), and the use of new combined treatments has been suggested (Botros et al. 2000; De Clercq et al. 2000). Due to its ability to modulate schistosomiasis morbidity leading to a mild disease course, dexamethasone may be an important tool for improving the quality of life in combined therapies. 
Nowadays, more modern and efficient techniques are employed for the clinical evaluation of schistosomiasis (Abdel-Wahab and Strickland 1993; Kariuki et al. 2001). However, due to its simplicity and low cost, the hematological and visceral parameters analyzed in this paper are still commonly used as clinical parameters in epidemiological studies (Kongs et al. 1996 ; Guyatt et al. 2001). Thus, the data presented here corroborate our previous results (Pyrrho et al. 2002), indicating that dexamethasone decreases the severity of schistosomiasis infection, and reinforces the suggestion of a beneficial use of immunomodulators as co-adjuvants in specific treatment of $S$. mansoni infections. Indeed, in Brazil the National Schistosomiasis Control Programs, which include early chemotherapy and other complementary measures, have led to a reduction in the diagnosis of patients in the advanced hepatosplenic stage of the disease (Andrade and Bina 1985). Since (1) there is a great similarity in granuloma development in the infection phases in mice and humans (Raso and Bogliolo 1970), (2) most of the patients are detect at an early phase of infection, and (3) re-infection is a very common occurrence in endemic areas, the use of dexamethasone as suggested here could be a beneficial possibility in Brazil as well as in some other countries. This suggestion implies the development and application of adequate protocols for patients, considering that human schistosomiasis is different from experimental models due to Symmers' pipe-stem fibrosis (Andrade 1965).

Acknowledgements This work was supported by FAPERJ, CNPq, FUJB, and FIOCRUZ. The authors are grateful to Dr. L. Correa for the Schistosoma mansoni cercariae. All experimental procedures were conducted in accordance with the Guidelines for Care and Use of Laboratory Animals (CAUAP) of the Institute of Biophysics Carlos Chagas Filho, which conform to the National Institute of Health (Bethesda, MD, USA) guidelines.

\section{References}

Abdel-Wahab MF, Strickland GT (1993) Abdominal ultrasonography for assessing morbidity from schistosomiasis. 2. Hospital studies. Trans R Soc Trop Med Hyg 87:135-137

Adewusi OI, Nix NA, Lu X, Colley DG, Secor WE (1996) Schistosoma mansoni: relationship of tumor necrosis factor-alpha to morbidity and collagen deposition in chronic experimental infection. Exp Parasitol 84:115-123

Andrade ZA (1962) Aspectos experimentais da esplenomegalia da esquistossomose. Rev Inst Med Trop SP 4:249-255

Andrade ZA (1965) Hepatic schistosomiasis: morphological aspects. In: Popper HS (ed) Progess in liver disease. Grune and Stratton, New York, pp 228-242

Andrade ZA, Bina JC (1983) The pathology of the hepatosplenic form of schistosomiasis mansoni in its advanced form (study of 232 complete necropsies). Mem Inst Oswaldo Cruz 78:285-305

Andrade ZA, Bina JC (1985) The changing pattern of pathology due to Schistosoma mansoni infection. Mem Inst Oswaldo Cruz 80:363-366

Bagshawe A (1970) A comparative study of hypersplenism in reactive and congestive splenomegaly. Br J Haematol 19:729737
Bina JC, Prata A (1983) Reversão da hepatoesplenomegalia pelo tratamento específico da esquistossomose. Rev Soc Bras Med Trop 10:214-218

Borojevic R, Santos da Silva C, Carvalho EA (1983) Chronic schistosomiasis mansoni: splenic myelopoiesis and inhibition of neutrophil granulocytopoiesis mediated by the sera of patients. J Infect Dis 148:422-426

Bosshardt SC, Freeman GLJr, Secor WE, Colley DG (1997) IL-10 deficit correlates with chronic, hypersplenomegaly syndrome in male CBA/J mice infected with Schistosoma mansoni. Parasite Immunol 19:347-353

Botros SS, Makary EA, Ahmed KM, Ibrahim AM, Nashed NN, El-Nahal HM, Doughty BL, Hassanein HI (2000) Effect of combined praziquantel and recombinant glutathione S-transferase on resistance to reinfection in murine schistosomiasis mansoni. Int $\mathbf{J}$ Immunopharmacol 22:979-988

Cheever AW, Dunn MA, Dean DA, Duvall RH (1983) Differences in hepatic fibrosis in ICR, $\mathrm{C} 3 \mathrm{H}$, and $\mathrm{C} 57 \mathrm{BL} / 6$ mice infected with Schistosoma mansoni. Am J Trop Med Hyg 32:1364-1369

Cheever AW, Duvall RH, Hallack TAJr, Minker RG, Malley JD, Malley KG (1987) Variation of hepatic fibrosis and granuloma size among mouse strains infected with Schistosoma mansoni. Am J Trop Med Hyg 37:85-97

Cheever AW, Jankovic D, Yap GS, Kullberg MC, Sher A, Wynn TA (1998) Role of cytokines in the formation and downregulation of hepatic circumoval granulomas and hepatic fibrosis in Schistosoma mansoni-infected mice. Mem Inst Oswaldo Cruz 93 [Suppl 1]:25-32

Cheever AW, Lenzi JA, Lenzi HL, Andrade ZA (2002) Experimental models of Schistosoma mansoni infection. Mem Inst Oswaldo Cruz 97:917-940

De Clercq D, Vercruysse J, Verle P, Kongs A, Diop M (2000) What is the effect of combining artesunate and praziquantel in the treatment of Schistosoma mansoni infections? Trop Med Int Health 5:744-746

Dolber PC, Spach MS (1993) Conventional and confocal fluorescence microscopy of collagen fibers in the heart. J Histochem Cytochem 41:465-469

Druilhe P, Hagan P, Rook GA (2002) The importance of models of infection in the study of disease resistance. Trends Microbiol 10:S38-46

Eberl M, Langermans JA, Frost PA, Vervenne RA, Van Dam GJ, Deelder AM, Thomas AW, Coulson PS, Wilson RA (2001) Cellular and humoral immune responses and protection against schistosomes induced by a radiation-attenuated vaccine in chimpanzees. Infect Immun 69:5352-5362

Fanning MM, Peters PA, Davis RS, Kazura JW, Mahmoud AA (1981) Immunopathology of murine infection with Schistosoma mansoni: relationship of genetic background to hepatosplenic disease and modulation. J Infect Dis 144:148-153

Garba A, Tohon Z, Sidiki A, Chippaux JP, De Chabalier F (2001) Efficacity of praziquantel in school-aged children in a hyperendemic zone for Schistosoma haematobium (Niger, 1999). Bull Soc Pathol Exot 94:42-45

Guyatt HL, Brooker S, Kihamia CM, Hall A, Bundy DA (2001) Evaluation of efficacy of school-based anthelmintic treatments against anaemia in children in the United Republic of Tanzania. Bull World Health Organ 79:695-703

Hagan P, Ndhlovu P, Dunne D (1998) Schistosome immunology: more questions than answers. Parasitol Today 14:407-412

Henderson GS, Nix NA, Montesano MA, Gold D, Freeman GLJr, McCurley TL, Colley DG (1993) Two distinct pathological syndromes in male CBA/J inbred mice with chronic Schistosoma mansoni infections. Am J Pathol 142:703-714

Homeida MA, El Tom I, Nash T, Bennett JL (1991) Association of the therapeutic activity of praziquantel with the reversal of Symmers' fibrosis induced by Schistosoma mansoni. Am J Trop Med Hyg 45:360-365

Jamra M, Maspes V, Meira DA (1964) Types and mechanisms of anemia in schistosomiasis mansoni. Rev Inst Med Trop SP 6:126-136 
Kariuki HC, Mbugua G, Magak P, Bailey JA, Muchiri EM, Thiongo $\mathrm{FW}$, King $\mathrm{CH}$, Butterworth AE, Ouma JH, Blanton RE (2001) Prevalence and familial aggregation of schistosomal liver morbidity in Kenya: evaluation by new ultrasound criteria. J Infect Dis 183:960-966.

Kongs A, Verle P, Dieng A, Talla I, Rouquet P (1996) Clinical investigation of a population recently infected with Schistosoma mansoni (Richard-Toll, Senegal). Trop Med Int Health 1:191198

Lambertucci JR, Modha J, Curtis R, Doenhoff M (1989) The association of steroids and schistosomicides in the treatment of experimental schistosomiasis. Trans R Soc Trop Med Hyg $83: 354-357$

Lambertucci JR, Rayes AA, Serufo JC, Nobre V (2001) Pyogenic abscesses and parasitic diseases. Rev Inst Med Trop Sao Paulo 43:67-74

Pyrrho AS, Ramos JA, Neto RM, Silva CS, Lenzi HL, Takiya CM, Gattass CR (2002) Dexamethasone, a drug for attenuation of Schistosoma mansoni infection morbidity. Antimicrob Agents Chemother 46:3490-3498

Raso P, Bogliolo L (1970) Patologia. In: Cunha ASD (ed) Esquistossomose mansoni. Sarvier and USP, São Paulo, pp 77130
Ross AG, Bartley PB, Sleigh AC, Olds GR, Li Y, Williams GM, McManus DP (2002) Schistosomiasis. N Engl J Med 346:12121220

Santos da Silva C, Carvalho EA, Goncalvez MS, Borojevic R (1988) Experimental murine schistosomiasis mansoni: inhibition of neutrophil granulocyte inflammatory reaction. Braz $\mathbf{J}$ Med Biol Res 21:273-279

Utzinger J, N'Goran EK, N'Dri A, Lengeler C, Tanner M (2000) Efficacy of praziquantel against Schistosoma mansoni with particular consideration for intensity of infection. Trop Med Int Health 5:771-778

Warren KS, Domingo EO, Cowan RB (1967) Granuloma formation around schistosome eggs as a manifestation of delayed hypersensitivity. Am J Pathol 51:735-756

Woodruff AW, Shafei AZ, Awwad HK, Pettitt LE, Abaza HH (1966) Anaemia in patients with schistosomiasis and gross splenomegaly. Trans R Soc Trop Med Hyg 60:343-351

World Health Organisation (1997) Schistosomiasis. Tropical Disease Research: Progress 1995-1996, 13th Programme Report of the UNDP/World Bank/WHO Special Programme for Research and Training in Tropical Diseases. World Health Organisation, Geneva, pp 62-73 